\title{
Identification of protein functions using a machine-learning approach based on sequence-derived properties Bum Ju Lee ${ }^{1}$, Moon Sun Shin², Young Joon Oh${ }^{1}$, Hae Seok $\mathrm{Oh}^{3}$ and Keun Ho Ryu*4
}

\author{
Address: ${ }^{1}$ Industrial Research Center, Jungwon University, 5 Dongbu-ri, Goesan-eup, Goesan-gun, Chungbuk 367-805, Republic of Korea, ${ }^{2}$ Dept. \\ of Computer Science, Konkuk University, 322 Danwol-Dong, Chungju-Si, Chungbuk 380-701, Republic of Korea, ${ }^{3}$ Dept. of Computer Science, \\ Kyungwon University, 161 Bokjung-Dong, Soojung-Gu, Seongnam-Si, Gyeonggi-Do 461-701, Republic of Korea and ${ }^{4}$ School of Electrical and \\ Computer Engineering, Chungbuk National University, 12 Gaeshindong, Cheongju, Chungbuk 361-763, Republic of Korea \\ Email: Bum Ju Lee - jupiter-lee@hanmail.net; Moon Sun Shin - msshin@kku.ac.kr; Young Joon Oh - drpro@jwu.ac.kr; \\ Hae Seok Oh - oh@kyungwon.ac.kr; Keun Ho Ryu* - khryu@dblab.chungbuk.ac.kr \\ * Corresponding author
}

Published: 9 August 2009

Proteome Science 2009, 7:27 doi:10.1/86/1477-5956-7-27

This article is available from: http://www.proteomesci.com/content/7///27

This is an Open Access article distributed under the terms of the Creative Commons Attribution License (http://creativecommons.org/licenses/by/2.0), which permits unrestricted use, distribution, and reproduction in any medium, provided the original work is properly cited.

Received: 13 March 2009

Accepted: 9 August 2009

\begin{abstract}
Background: Predicting the function of an unknown protein is an essential goal in bioinformatics. Sequence similarity-based approaches are widely used for function prediction; however, they are often inadequate in the absence of similar sequences or when the sequence similarity among known protein sequences is statistically weak. This study aimed to develop an accurate prediction method for identifying protein function, irrespective of sequence and structural similarities.

Results: A highly accurate prediction method capable of identifying protein function, based solely on protein sequence properties, is described. This method analyses and identifies specific features of the protein sequence that are highly correlated with certain protein functions and determines the combination of protein sequence features that best characterises protein function. Thirty-three features that represent subtle differences in local regions and full regions of the protein sequences were introduced. On the basis of 484 features extracted solely from the protein sequence, models were built to predict the functions of II different proteins from a broad range of cellular components, molecular functions, and biological processes. The accuracy of protein function prediction using random forests with feature selection ranged from $94.23 \%$ to $100 \%$. The local sequence information was found to have a broad range of applicability in predicting protein function.
\end{abstract}

Conclusion: We present an accurate prediction method using a machine-learning approach based solely on protein sequence properties. The primary contribution of this paper is to propose new PNPRD features representing global and/or local differences in sequences, based on positively and/ or negatively charged residues, to assist in predicting protein function. In addition, we identified a compact and useful feature subset for predicting the function of various proteins. Our results indicate that sequence-based classifiers can provide good results among a broad range of proteins, that the proposed features are useful in predicting several functions, and that the combination of our and traditional features may support the creation of a discriminative feature set for specific protein functions. 


\section{Background}

The need to analyse the massive accumulation of biological data generated by high-throughput human genome projects has stimulated the development of new and rapid computational methods. Computational approaches for predicting and classifying protein functions are essential in determining the functions of unknown proteins in a faster and more cost-effective manner, because experimentally determining protein function is both costly and time-consuming. Approaches based on sequence and structure comparisons play an important role in predicting and classifying the function of unknown proteins. Generally, if an unknown gene or protein sequence is identified, researchers may carry out a sequence similarity search using BLAST [1], PSI-BLAST [2], or FASTA [3] to find similar proteins or annotation information in public databases. However, proteins that have diverged from a common ancestral gene may have the same function but different sequences $[4,5]$. As a result, sequence similaritybased approaches are often inadequate in the absence of similar sequences or when the sequence similarity among known protein sequences is statistically weak (called the "twilight zone" or "midnight zone") [6-12]. Thus, researchers should be cautious when using this approach, because its efficiency is limited by the availability of annotated sequences in public databases and a high-similarity BLAST search result does not always imply homology $[13,14]$. Structure similarity-based approaches - such as Deli[15] or MATRAS [16], both of which use structure comparisons - have routinely been used to identify proteins with similar structures, because protein structural information is better conserved than sequence information $[17,18]$. Nevertheless, proteins with the same function can have different structures, and a structural comparison through structure determination is more difficult than a sequence comparison $[9,19,20]$.

Recently, several researchers have developed methods for classifying and predicting protein function independent of sequence or structural alignment [5-9,20-50]. Rather than making predictions based on direct sequence or structural comparisons, these approaches use various features to predict protein function, such as protein length, molecular weight, number of atoms, grand average of hydropathicity (GRAVY), amino acid composition, periodicity, physicochemical properties, predicted secondary structures, subcellular location, sequence motifs or highly conserved regions, and annotations in protein databases. These features include statistics extracted from the protein sequence, structure, and annotation. In addition, to obtain good predictive power, various machine-learning algorithms such as support vector machines (SVMs), neural networks, naïve Bayes classifiers, and ensemble classi- fiers have been used to build classification and prediction models. Of these, the most widely used machine-learning algorithm for classification and prediction of protein function is SVM $[5-7,22,26,27,29,31,36,39-43,45]$.

A method for classifying the functions of homodimeric, drug absorption, drug delivery, drug excretion, and RNAbinding proteins, among others, has been proposed by Cai et al. [29]. Classification of protein function was performed using an SVM statistical learning algorithm, based on features such as amino acid composition, hydrophobicity, solvent accessibility, secondary structure, surface tension, charge, polarisability, polarity, and normalised van der Waals volume. Cai et al. [29] found that the testing accuracy of protein classification was in the range $84-96 \%$ and suggested that amino acid composition, hydrophobicity, polarity, and charge play more critical roles than other features. Recently, Tung et al. [40] proposed a prediction method for ubiquitylation sites using three datasets (amino acid identity, physicochemical properties, and evolutionary information) and three machine-learning algorithms (k-nearest neighbour, SVM, and naïve Bayes). The greatest accuracy $(72.19 \%)$ was obtained using SVM with 531 physicochemical properties as features. Moreover, accuracy improved from $72.19 \%$ to $84.44 \%$ when 31 physicochemical properties were selected and used based on feature selection by an informative physicochemical property mining algorithm (IPMA). In addition, Li et al. [45] demonstrated the ability of the SVM prediction method to identify potential drug targets. On the basis of amino acid composition, hydrophobicity, polarity, polarisability, charge, solvent accessibility, and normalised van der Waals volume, they obtained an accuracy of $84 \%$ in predicting known drug targets versus putative nondrug targets. In that study, the performance of the SVM model did not change significantly with a greater number of negative targets, as determined from experiments using various ratios of negative to positive samples.

Protein function has been predicted using naïve Bayes classifiers in several studies $[21,49,50]$. The FEATURE framework for recognition of functional sites in macromolecular structures was developed by Halperin et al. [49]. They used naïve Bayes classification to find and weigh the most informative properties that distinguish sites from nonsites, using a large number of physicochemical properties. In another study, Gustafson et al. [50] suggested a classification method for identification of genes essential to survival by using naïve Bayes classification. They focused on easily obtainable features such as open reading frame (ORF) size, upstream size, phyletic retention, amino acid composition, codon bias, and hydro- 
phobicity, and they concluded that the best performing feature was phyletic retention or the presence of an orthologue in other organisms.

Neural networks are also frequently used for function prediction $[23-25,36]$. For example, six enzyme classes and enzymes/nonenzymes were predicted by Jensen et al. [24], based solely on a few meaningful features such as $\mathrm{O}-\beta$ GlcNAcsites, N-linked glycosylation, secondary structure, and physicochemical properties. Function prediction was carried out using a neural network, and certain meaningful features - such as differences in secondary structures between enzymes and nonenzymes - were analysed. The discriminative ability of each feature was represented by its correlation coefficient.

Ensemble classifiers have recently become popular for protein function classification $[25,35,46,47,51-55]$. Zhao et al. [35] suggested that no single-classifier method can always outperform other methods and that ensemble classifier methods outperform other classifier methods because they use various types of complementary information. In comparing the performance of classifiers for predicting glycosylation sites, Caragea et al. [52] demonstrated that ensembles of SVM classifiers outperformed single SMV classifiers. In addition, Guan et al. [51] illustrated the benefits of using an SVM-based ensemble framework by analysing the performance of ensembles of three classifiers as a single SVM, a hierarchically corrected combination of SVMs, and naïve Bayes classifiers. Ge et al. [53] provided evidence that ensemble classifiers outperform single decision tree classifiers by comparing C4.5 with several ensemble classifiers (i.e. random forest, stacked generalisation, bagging, AdaBoost, LogitBoost, and MultiBoost) for classification of premalignant pancreatic cancer mass-spectrometry data. A prediction method using a domain-based random forest of decision trees to infer protein-protein interactions (PPIs) was proposed by Chen et al. [46]; in experiments using a Saccharomyces cerevisiae dataset, they showed that the randomforest method achieved higher sensitivity $(79.78 \%)$ and specificity (64.38\%) than maximum likelihood estimation (MLE).

These previous studies exploit direct relationships between basic protein properties and their functions to predict protein function without consideration of sequence or structural similarities. Among these studies, a wide variety of features or only a few meaningful features were selected to increase the performance of function prediction based on experience or a few heuristics. However, in most of the previous studies, features that represent subtle distinctions in small portions of protein sequences have not been sufficiently represented. Although proteins share similar structural organisations, biological properties, and sequences, small changes in amino acids of a protein sequence can result in different functions $[19,20,56]$. Although local information such as the presence of motifs or highly conserved regions is useful for function prediction, motif detection problems present another arduous task. Therefore, in this study, a method was developed to detect small changes in amino acids within a sequence.

The method described here is characterised by three primary features designed to address specific problems inherent in protein function prediction. First, this approach was designed to accurately predict various protein functions over a broad range of cellular components, molecular functions, and biological processes without using sequence or structural similarity information. Second, this study was designed to determine whether the use of feature selection improves prediction performance for various protein functions. In other words, does the use of more features related to the protein sequence increase the accuracy of prediction? Third, this study was designed to determine whether local information for the protein sequence is meaningful in predicting protein function, and if so, to determine which features are correlated with protein function.

In summary, a highly accurate prediction method capable of identifying protein function is proposed. One of the advantages of this method is that it requires only the protein sequence for feature extraction; information vis-à-vis predicted features or structural properties is not required. In addition, four features that represent subtle differences in local regions of the protein sequence - differences due to positively and negatively charged residues - are introduced. A total of 484 features, including 451 traditional features and 33 features introduced in this study, were used to predict 11 protein functions. We applied two machine-learning algorithms (i.e. SVM and random forests) with and without feature selection to the data set to predict protein function. The prediction performance for each protein function was evaluated, and the features most relevant to prediction of specific protein functions were determined.

\section{Methods \\ Data preparation}

To predict the functions of a variety of proteins from a broad range of cellular components, molecular functions, and biological processes, 16,618 positive sample sequences and 35,619 negative sample sequences were collected and comprised the dataset. The dataset included positive and negative samples for 11 protein functions selected from the Swiss-Prot database [57] using the SRS program [58]. Positive samples are sample sequences associated with a specific protein function and were labelled as belonging to that class of proteins. Negative samples for each protein class were selected from proteins 
that do not belong to that class and from enzyme families such as oxidoreductases, hydrolases, lyases, and isomerases. For example, the composition of the negative sample set for fatty acid metabolism is shown in Table 1. Proteins that consisted of fewer than 30 amino acids were excluded from the dataset. In the feature extraction step, proteins that had missing values were also excluded. For hypothetically or automatically annotated sequences in protein databases such as GenBank and Swiss-Prot, the percentage of incorrect annotations is not known, because the annotations do not include a description of the specific methodology used for sequence analysis; this sometimes yields incorrect search results [14]. However, Swiss-Prot incorporates corrections provided by user forums and communities [13]; therefore, protein sequences from the SwissProt database were used in the present study.

\section{Feature extraction from protein sequences}

A total of 484 features were extracted solely from the protein sequences described in this study. These features included traditional features adopted from previous studies $[7,30-32]$ and new features extracted using the novel method developed in this study. Among the traditional features, 34 were extracted from the Swiss-Prot protein sequences [57] using the ProtParam tool [59]. Traditional features consisted of amino acid composition, protein length, number of atoms, molecular weight, GRAVY, and theoretical isoelectric point (pI), among others. In addition, two ways of using positively charged residues (i.e. lysine and arginine or histidine, lysine, and arginine), the percent composition of each amino acid pair, and 17 features based on physicochemical properties (i.e. 16 properties adopted from Syed et al. [20] and one additional property) were calculated.

The importance of negatively/positively charged residues in protein function has been described in several studies

Table I: Negative samples for the fatty acid metabolism protein class

\begin{tabular}{lc}
\hline Protein class & Number of proteins \\
\hline Transport & 637 \\
Transcription & 538 \\
Gluconate utilisation & 60 \\
Amino acid biosynthesis & 393 \\
DNA-binding & 486 \\
Acetylcholine receptor inhibitor & 103 \\
G-protein coupled receptor & 220 \\
Guanine nucleotide-releasing factor & 370 \\
Fibre protein & 47 \\
Transmembrane & 351 \\
Oxidoreductases & 58 \\
Hydrolases & 75 \\
Isomerases & 50 \\
Lyases & 72 \\
Other proteins & 354 \\
\hline
\end{tabular}

$[20,23,24,38,60]$. The 20 standard amino acids are divided into negatively charged residues, positively charged residues, and neutral residues according to their pI. Negatively charged residues (aspartic acid and glutamic acid) have lower pIs, while positively charged residues (arginine and lysine) have higher pIs. Oppositely charged residues attract, while similarly charged residues repel each other. To account for subtle differences that occur in small regions of the protein sequences, features representing the percentage change in charged residues as well as the distribution of charged residues were designed and computed.

The method used to identify these new features is simple. $P P R$ was calculated using the following equation:

$$
P P R=\frac{(\# P P-1)}{\# A A} \times 100
$$

where \#AA is the total number of amino acids in a sequence and \#PP is the total number of continuous changes from one positively charged residue to the next positively charged residue in each protein sequence. Similar to PPR, NNR was calculated using the following equation:

$$
N N R=\frac{(\# N N-1)}{\# A A} \times 100
$$

where $\# N N$ is the total number of continuous changes from one negatively charged residue to the next negatively charged residue. PNPR was calculated using the following equation:

$$
P N P R=\frac{(\# P N P-1)}{\# A A} \times 100
$$

where \#PNP is the total number of continuous changes from a positively charged residue to the next negatively charged residue or vice versa. Finally, Dist $(x, y)$ is the distribution function for $P P, N N$, or $P N P$ in the interval from $x$ to $y$ in the sequence, with the stipulation that $x<y$. PPRDist $t_{(x, y)}$ was defined as follows:

$$
\operatorname{PPRDist}_{(x, y)}=\frac{\# P P(x, y)}{\# A A} \times 100
$$

where $\# P P_{(x, y)}$ is the total number of $P P$ occurrences in the interval from $x$ to $y$. Similarly, NNRDist $(x, y)$ was computed as follows:

$$
\operatorname{NNRDist}_{(x, y)}=\frac{\# N N(x, y)}{\# A A} \times 100
$$


where $\# N N_{(x, y)}$ is the total number of NN occurrences in the interval from $x$ to $y$.PNPRDist $(x, y)$ was computed as follows:

$$
\operatorname{PNPRDist}_{(x, y)}=\frac{\# P N P(x, y)}{\# A A} \times 100
$$

where $\# P N P_{(x, y)}$ is the total number of PNP occurrences of the interval from $x$ to $y$. These features provide local information on a protein sequence based on the values of $x$ and $y$. For example, the alcohol dehydrogenase1A protein (Swiss-Prot:P07327) consists of 375 amino acids. Let us assume that the $x$ value is the 76th amino acid (21\%), the $y$ value is the 113th amino acid (30\%), and the value of PNP is 4. PNPRDist $(21,30)$ is thus $(4 / 375) \times 100=1.06667$. $^{2}$ We believe these features are important because slight regional differences among similar proteins exist in sequences within the same family. Certain protein functions are determined by a few residues within a small part of the sequence [61]. A total of 33 features were generated based on the above formulae, dividing the sequence length into 10 local regions, specifically, PPR (1), NNR (1), PNPR (1), PPRDist $(x, y)$ (10), NNRDist $(x, y)$ (10), and PNPRDist $_{(x, y)}(10)$. All the traditional and novel features used in this study are described in detail in Table 2.

\section{Feature selection}

Feature selection is an important step in developing an accurate classification method. There are many redundant and/or irrelevant features in real-world problems, and various approaches have been developed to address these features. The primary goals of feature selection [62-65] are to gain a more thorough understanding of the underlying processes influencing the data and to identify discriminative and useful features for classification and prediction. In addition, classification and prediction performance can be improved by avoiding overfitting. Although additional features provide more information and could potentially improve classification performance, a greater number of features also adds difficulty in building a classifier. For $n$ features there are $n^{2}$ possible feature subsets; therefore, to achieve optimal performance, it is necessary to generate all possible subsets and examine their performance.

Various feature selection methods have been developed to select an optimal feature set and analyse the discriminatory power of each feature. Feature subset selection techniques can be organised into two categories: filter and wrapper methods. Filter methods, which apply statistical approaches without any information on the classification algorithm, are used to select a specific subset of potentially discriminating features. Wrapper methods use a machine-learning algorithm, called a perfect "black box," to assess the quality of a feature subset. For this study, correlation-based feature selection (CFS) $[34,65]$ was used to select a subset of discriminative features. CFS was chosen for the following reasons. First, when the number of features is large, filter methods are faster than wrapper methods because the former do not require the use of learning machines. In addition, filter methods can be used as a preprocessing step to reduce space dimensionality and preclude overfitting. Second, selection and evaluation of a subset of features is preferable to individually important features because a superior classifier can be constructed from features that interact or by a combination of many features that together have discriminatory power. Even if one or two features are not useful alone, these features may be valuable in combination with other features and thus improve the discriminatory performance of a classifier [64].

CFS is a filter method. It uses a search algorithm, along with a function for evaluating the merit of a feature subset, based on the hypothesis that "a good feature subset contains features highly correlated with the class, yet uncorrelated with each other" [65]. This method evaluates subsets of features, rather than individual features, as discussed above. At the core of the CFS is the subset evaluation heuristic. It eliminates irrelevant features, as they will be poor predictors of classes. In addition, redundant features are identified that will be highly correlated with one or more other features. A heuristic search to traverse the space of the feature set is conducted, and the subset with the highest merit found during the search process is reported. The subset with the highest merit preserves the most important features - those that are highly correlated with the class and have low inter-correlation with one another. This subset is then used to reduce dimensionality. CFS is described in greater detail elsewhere [65].

In the present study, many features were discarded during the feature subset selection procedure using CFS. Merit was calculated using the following equation:

$$
\text { Merit }_{s}=\frac{k \overline{r_{c f}}}{\sqrt{k+k(k-1) \overline{r_{f f}}}}
$$

Where Merit is the score of a feature subset $S$ that comprises $k$ features, $\overline{r_{c f}}$ is the average correlation between the individual features and the class, and $\overline{r_{f f}}$ is the average inter-correlation among the features. The features selected by CFS for each class are listed in Table 3.

Finding and identifying important features that discriminate protein function is an arduous task; however, it is possible to evaluate which discriminative features are important using feature subset selection methods. For 
Table 2: Features used for protein function classification

\begin{tabular}{|c|c|c|c|}
\hline & Feature & Description & Dimension \\
\hline I & Number of amino acids & Number of residues in each protein & I \\
\hline 2 & Molecular weight & Molecular weight of the protein & I \\
\hline 3 & Theoretical pl & The $\mathrm{pH}$ at which the net charge of the protein is zero (isoelectric point) & I \\
\hline 4 & Amino acid composition & Percentage of each amino acid in the protein & 20 \\
\hline 5 & Positively charged residue_2 & Percentage of positively charged residues in the protein (lysine and arginine) & I \\
\hline 6 & Positively charged residue_3 & Percentage of positively charged residues in the protein (histidine, lysine, and arginine) & I \\
\hline 7 & Number of atoms & Total number of atoms & I \\
\hline 8 & Carbon & Total number of carbon atoms in the protein sequence & I \\
\hline 9 & Hydrogen & Total number of hydrogen atoms in the protein sequence & I \\
\hline 10 & Nitrogen & Total number of nitrogen atoms in the protein sequence & I \\
\hline II & Oxygen & Total number of oxygen atoms in the protein sequence & I \\
\hline 12 & Sulphur & Total number of sulphur atoms in the protein sequence & I \\
\hline 13 & Extinction coefficient_All & $\begin{array}{l}\text { Amount of light a protein absorbs at a certain wavelength } \\
\text { (assuming ALL Cys residues appear as half cysteines) }\end{array}$ & I \\
\hline 14 & Extinction coefficient_No & $\begin{array}{l}\text { Amount of light a protein absorbs at a certain wavelength } \\
\text { (assuming NO Cys residues appear as half cysteines) }\end{array}$ & I \\
\hline 15 & Instability index & The stability of the protein & I \\
\hline 16 & Aliphatic index & The relative volume of the protein occupied by aliphatic side chains & I \\
\hline 17 & GRAVY & Grand average of hydropathicity & I \\
\hline 18 & PPR & $\begin{array}{l}\text { Percentage of continuous changes from positively charged residues to positively charged } \\
\text { residues }\end{array}$ & I \\
\hline 19 & NNR & $\begin{array}{l}\text { Percentage of continuous changes from negatively charged residues to negatively charged } \\
\text { residues }\end{array}$ & I \\
\hline 20 & PNPR & $\begin{array}{l}\text { Percentage of continuous changes from positively charged residues to negatively charged } \\
\text { residues or from negatively charged residues to positively charged residues }\end{array}$ & I \\
\hline 21 & NNRDist $_{(x, y)}$ & Percentage of NNR from $x$ to $y$ (local information) & 10 \\
\hline 22 & PPRDist $_{(x, y)}^{(x, y)}$ & Percentage of PPR from $x$ to $y$ (local information) & 10 \\
\hline 23 & PNPRDist $_{(x, y)}$ & Percentage of PNPR from $x$ to $y$ (local information) & 10 \\
\hline 24 & Charged & Physicochemical property & I \\
\hline 25 & Negatively charged residues & Percentage of negatively charged residues in the protein & I \\
\hline 26 & Polar & Physicochemical property & I \\
\hline 27 & Aliphatic & Physicochemical property & I \\
\hline 28 & Aromatic & Physicochemical property & I \\
\hline 29 & Small & Physicochemical property & I \\
\hline 30 & Tiny & Physicochemical property & I \\
\hline 31 & Bulky & Physicochemical property & I \\
\hline 32 & Hydrophobic & Physicochemical property & I \\
\hline 33 & Hydrophobic and aromatic & Physicochemical properties & I \\
\hline 34 & $\begin{array}{l}\text { Neutral, weakly and } \\
\text { hydrophobic }\end{array}$ & Physicochemical properties & I \\
\hline 35 & Hydrophilic and acidic & Physicochemical properties & I \\
\hline 36 & Hydrophilic and basic & Physicochemical properties & I \\
\hline 37 & Acidic & Physicochemical property & I \\
\hline 38 & Polar and uncharged & Physicochemical properties & I \\
\hline \multirow[t]{2}{*}{39} & Amino acid pair ratio & Percentage compositions for each of the 400 possible amino acid dipeptides & 400 \\
\hline & Total & & 484 \\
\hline
\end{tabular}

instance, using the CFS method, for the transmembrane protein class (Table 4 , last row), the number of traditional features selected was 33 of 451 features and the number of new features selected was 3 of 33 features. Selection rates were then calculated as $(33 / 451) \times 100=7.76 \%$ and $(3 /$ $33) \times 100=9.09 \%$. Several of the new features were preserved in every subset selected by the CFS method, except for the transport class. Therefore, it can be inferred that these features are highly correlated with the class and that they have low inter-correlation with each other.

\section{Support vector machines and random forests}

In the preprocessing step, numeric features were discretised via an MDL-based discretisation method [66]. Each dataset was randomly split into a training set $(90 \%)$ and a blind test set (10\%). The numbers of negative and positive 
Table 3: Features selected by CFS for each protein class

\begin{tabular}{ll}
\hline Protein class & Selected features \\
\hline Transport & R, G, H, I, M, positively charged residue_3, carbon, CC, CD, CE, CH, CK, CN, CQ, CW, CY, FM, GW, HC, \\
HR, IC, IG, LF, LG, LM, MF, MM, MQ, PC, QC, SC, TC, WD, YH, polar, hydrophobic, hydrophobic and \\
aromatic, hydrophilic and basic
\end{tabular}

Translation

NumOfAAs, D, L, hydrogen, GRAVY, PPR, NNR, NNRD $(11,20), P P R D_{(31,40)}, \operatorname{PNPRD}_{(41,50)}, P P R D_{(51,60)}, \operatorname{PNPRD}_{(81,90)}$,

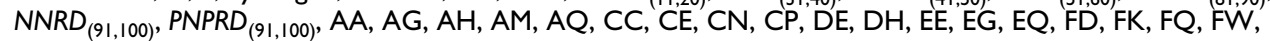
GC, GV, GW, GY, HI, IC, IP, IY, KE, KK, KR, KS, KW, LG, LK, LT, LV, LW, MM, MW, NH, PE, PK, PT, PY, QF, QN, RN, SD, TG, TK, VA, VG, VL, WC, WE, WG, WK, YD, YS, YV, charged, aliphatic, hydrophilic and acidic

Gluconate utilisation

Positively charged residue_3, instability index, aliphatic index, PNPRDist ${ }_{(1,20)}$, PPRDist $_{(21,30)}$, PPRDist $_{(31,40)}$, PPRDist $_{(81,90}$, PPRDist $_{(91,100)}, \mathrm{AG}, \mathrm{AH}, \mathrm{AV}, \mathrm{AW}, \mathrm{AY}, \mathrm{CC}, \mathrm{Cl}, \mathrm{DG}, \mathrm{DI}, \mathrm{DR}, \mathrm{EG}, \mathrm{EW}, \mathrm{FH}, \mathrm{FL}, \mathrm{FP}, \mathrm{GC}, \mathrm{GE}, \mathrm{GF}, \mathrm{GI}$, GK, GM, GP, GR, HN, IG, KL, KM, KW, LI, LM, MG, MM, MQ, MV, PC, PK, PN, PP, SR, SY, TD, VF, VM, WN, WR, WT, YS, YV, aromatic, hydrophilic and acidic, polar and uncharged

Amino acid biosynthesis NumOfAAs, theoretical pl, D, C, G, S, sulphur, instability index, aliphatic index, GRAVY, PPR, NNR,

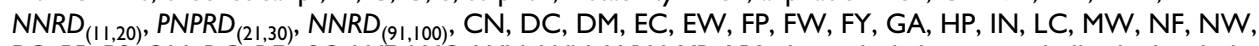
PC, PP, PS, QM, RC, RD, SC, WF, WG, WM, WN, WW, YR, YY, charged, aliphatic, tiny, bulky, hydrophobic, hydrophobic and aromatic, hydrophilic and acidic, acidic

\begin{tabular}{|c|c|}
\hline Fatty acid metabolism & 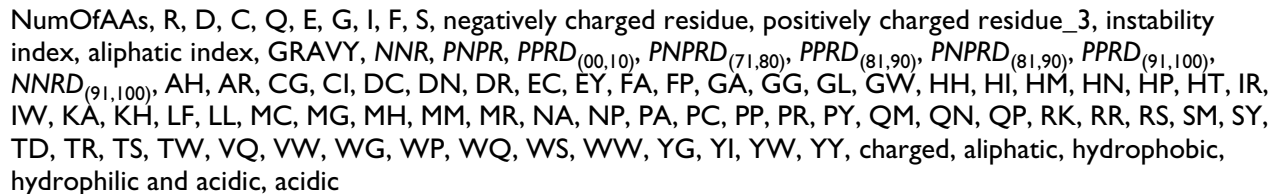 \\
\hline
\end{tabular}

Acetylcholine receptor inhibitor

Molecular weight, C, M, PNPRDist $(00,10)$, NNRDist $_{(1,20)}$, NNRDist $_{(71,80)}, \mathrm{AN}, \mathrm{AT}, \mathrm{CA}, \mathrm{CC}, \mathrm{CF}, \mathrm{CN}, \mathrm{CP}, \mathrm{CS}, \mathrm{DA}$, DF, DP, DS, EA, EI, ES, ET, FL, GC, HI, HQ, IC, II, IR, IT, KC, KE, KF, KL, KT, LD, LE, LN, LP, LQ, MK, NC, $\mathrm{NV}, \mathrm{RI}, \mathrm{TC}, \mathrm{VK}, \mathrm{VN}, \mathrm{VS}, \mathrm{WC}, \mathrm{YD}, \mathrm{YT}$, tiny

G-protein coupled receptor

Theoretical pl, D, C, Q, E, G, K, F, S, T, negatively charged residue, positively charged residue_3, sulphur, PNPR, PNPRDist $(11,20)$, NNRDist $_{(71,80)}, \mathrm{CC}, \mathrm{CF}, \mathrm{CH}, \mathrm{CW}, \mathrm{CY}, \mathrm{FC}, \mathrm{FI}, \mathrm{FL}, \mathrm{GQ}, \mathrm{IC}, \mathrm{IW}, \mathrm{IY}, \mathrm{LC}, \mathrm{MW}, \mathrm{SC}, \mathrm{WG}$ $\mathrm{WV}, \mathrm{WY}$, aromatic, tiny, bulky, hydrophobic and aromatic, acidic
Guanine nucleotide-releasing factor $\mathrm{A}, \mathrm{Q}, \mathrm{H}, \mathrm{I}, \mathrm{V}$, positively charged residue_2, positively charged residue_3, oxygen, instability index, aliphatic index, GRAVY, PPR, NNR, NNRDist ${ }_{(00,10)}$, PPRDist $_{(11,20)}$, PNPRDist $_{(21,30)}$, NNRDist $_{(31,40)}$, PNPRDist $_{(51,60)}$, PNPRDist $_{(61,70)}$, NNRDist $_{(91,100)}, \mathrm{CQ}, \mathrm{DC}, \mathrm{DH}, \mathrm{EC}, \mathrm{ED}, \mathrm{EE}, \mathrm{EP}, \mathrm{EW}, \mathrm{FN}, \mathrm{HC}, \mathrm{HD}, \mathrm{HE}, \mathrm{HH}, \mathrm{HK}, \mathrm{HM}, \mathrm{HW}, \mathrm{IV}$, KW, LE, LG, LK, MF, MI, PN, QC, QD, QE, QW, RL, SE, SP, TW, VG, VI, VV, WC, WD, WE, WF, WK, WS, WY, YW, hydrophobic, hydrophilic and acidic, hydrophilic and basic, acidic, polar and uncharged, polar

Fibre protein G, M, T, positively charged residue_2, NNRDist ${ }_{(81,90)}$, DN, ER, FN, GD, GG, GN, GQ, GT, IN, IP, LC, LL, LT, NA, NG, NT, PF, SQ, TA, TG, TN, TW, WK, WN, charged, polar and uncharged

Transmembrane

Theoretical pl, D, C, L, S, W, negatively charged residue, extinction coefficient_all, instability index, GRAVY, NNR, PNPR, PPRDist $t_{(71,80)}, \mathrm{AD}, \mathrm{CC}, \mathrm{CW}, \mathrm{DA}, \mathrm{EA}, \mathrm{FC}, \mathrm{FL}, \mathrm{FW}, \mathrm{LK}, \mathrm{LL}, \mathrm{LW}, \mathrm{MW}, \mathrm{PC}, \mathrm{PP}, \mathrm{SC}, \mathrm{SL}, \mathrm{TW}, \mathrm{VD}$, WW, tiny, bulky, acidic

samples in the training and test data sets are shown in Table 5. Validation was performed by 10 -fold cross-validation on the training set, and test results for the blind test process were obtained using a separate test dataset. No sample was included in both the training and testing sets. We present only the average performance of the 10-fold cross-validation process because the Weka tool [67] does not provide experimental results for each iteration of $k$ fold cross-validation.

The abilities of the SVM and random forest techniques to predict and classify protein functions have recently been 
Table 4: Selection ratios for traditional and new features in the CFS method

\begin{tabular}{lcrr}
\hline Protein class & Number of selected features & $\begin{array}{r}\text { Merit value } \\
\text { Nransport }\end{array}$ & $\begin{array}{r}\text { Traditional features }(\mathrm{n}=45 \mathrm{I}) \\
\text { New features } \\
(\mathrm{n}=33)\end{array}$ \\
\hline Transcription & 38 & 0.302 & $0 \%$ \\
Translation & 51 & 0.387 & $8.43 \%$ \\
Gluconate utilisation & 76 & 0.499 & $11.31 \%$ \\
Amino acid biosynthesis & 59 & 0.59 & $16.85 \%$ \\
Fatty acid metabolism & 52 & 0.309 & $13.08 \%$ \\
Acetylcholine receptor inhibitor & 90 & 0.303 & $11.53 \%$ \\
G-protein coupled receptor & 52 & 0.974 & $15.15 \%$ \\
Guanine nucleotide-releasing factor & 39 & 0.487 & $15.15 \%$ \\
Fibre protein & 69 & 0.36 & $24.24 \%$ \\
Transmembrane & 31 & 0.481 & $9.09 \%$ \\
& 35 & 0.443 & $9.09 \%$ \\
\hline
\end{tabular}

The merit value is the highest merit calculated for an optimal subset of the features for each class. The selected features are highly correlated with the class and have low inter-correlation with each other.

enhanced and found to be superior to other classification algorithms $[5,6,22,26,29,33,46,47]$. SVM is essentially a two-class classifier, although the classifier can be extended to multiclass classifications. In this model, each object is mapped to a point in a high-dimensional space, where each dimension corresponds to a feature. The coordinates of the point are the frequencies of the features in their corresponding dimensions. In the training step, SVM learns the maximum-margin hyper-planes separating each class. In the testing step, a new object is classified by mapping it onto a point in the same high-dimensional space, divided by the hyper-plane that was learned in the training step.

Recently, the random forest method [68] has also become popular for protein function prediction. Random forests is a classification algorithm that employs an ensemble of

Table 5: Accuracy of predictions using training and blind test datasets with the SVM and random forest methods

\begin{tabular}{|c|c|c|c|c|c|c|c|c|c|c|c|c|c|}
\hline \multirow[t]{2}{*}{ Category } & \multirow[t]{2}{*}{ Protein class } & \multicolumn{2}{|c|}{ Training set } & \multicolumn{2}{|c|}{ Test set } & \multicolumn{2}{|c|}{ SVM_FF } & \multicolumn{2}{|c|}{ SVM_CFS } & \multicolumn{2}{|c|}{ RF_FF } & \multicolumn{2}{|c|}{ RF_CFS } \\
\hline & & Positive & Negative & Positive & Negative & Train & Test & Train & Test & Train & Test & Train & Test \\
\hline \multirow{6}{*}{$\begin{array}{l}\text { Biological } \\
\text { process }\end{array}$} & Transport & 2,824 & 3,583 & 298 & $4 \mid 4$ & 73.26 & 71.34 & 94.38 & 93.53 & 93.14 & 92.41 & 94.66 & 94.24 \\
\hline & Transcription & 3,644 & 3,872 & 415 & 421 & 87.78 & 85.04 & 96.62 & 96.65 & 94.25 & 94.61 & 94.65 & 94.73 \\
\hline & Translation & 139 & 1,886 & 16 & 210 & 98.81 & 98.67 & 98.37 & 97.78 & 97.87 & 96.90 & 98.07 & 97.34 \\
\hline & $\begin{array}{l}\text { Gluconate } \\
\text { utilisation }\end{array}$ & 53 & 420 & 7 & 46 & 98.73 & 98.11 & 98.94 & 98.11 & 97.04 & 98.11 & 98.30 & 100 \\
\hline & $\begin{array}{l}\text { Amino acid } \\
\text { biosynthesis }\end{array}$ & 2,769 & 3,970 & 289 & 460 & 73.55 & 76.63 & 90.28 & 92.12 & 95.69 & 96.12 & 96.29 & 96.12 \\
\hline & $\begin{array}{l}\text { Fatty acid } \\
\text { metabolism }\end{array}$ & 601 & 3,445 & 81 & 369 & 90.58 & 87.55 & 94.19 & 92 & 95.99 & 94.88 & 96.93 & 95.77 \\
\hline \multirow[t]{3}{*}{$\begin{array}{l}\text { Molecular } \\
\text { function }\end{array}$} & $\begin{array}{l}\text { Acetylcholine } \\
\text { receptor inhibitor }\end{array}$ & 93 & 1,840 & 10 & 205 & 100 & 99.53 & 100 & 100 & 100 & 100 & 100 & 100 \\
\hline & $\begin{array}{l}\text { G-protein coupled } \\
\text { receptor }\end{array}$ & $2,57 \mid$ & 3,828 & 263 & 448 & 76.04 & 77.07 & 98.76 & 98.17 & 96.62 & 97.74 & 97.60 & 97.46 \\
\hline & $\begin{array}{l}\text { Guanine nucleotide- } \\
\text { releasing factor }\end{array}$ & 335 & 3,994 & 35 & 446 & 98.96 & 98.75 & 99.51 & 98.96 & 98.49 & 98.96 & 98.98 & 98.54 \\
\hline $\begin{array}{l}\text { Cellular } \\
\text { component }\end{array}$ & Fibre protein & 42 & 1,266 & 6 & 140 & 99.84 & 99.31 & 99.92 & 99.31 & 99.38 & 99.31 & 99.84 & 98.63 \\
\hline Domain & Transmembrane & 1,904 & 3,930 & 223 & 426 & 80.01 & 79.81 & 97.15 & 97.84 & 96.02 & 97.84 & 96.46 & 97.38 \\
\hline
\end{tabular}

The accuracy of predictions using the training dataset was determined when building the classification model using 10 -fold cross validation, and the accuracy of predictions using the test dataset was determined using the built model. The accuracies of predictions for all the training and test datasets are presented to demonstrate a good balance between overfitting and underfitting. Positive: number of positive samples; negative: number of negative samples; FF: full features; CFS: correlation-based feature subset selection method. The bold values mean the highest values among four methods. 
classification trees that each use several bootstrap samples of training data and a randomly selected subset of features. The basic random forest method, using unpruned decision trees, selects features at random at each decision node. The final classification is obtained by combining the results of the trees via voting.

To identify protein functions in this study, LibSVM $[69,70]$ and random forests [68] (available at Weka [67]) were used as the classification algorithms. The type of SVM used was a C-SVC machine, and the kernel was a radial basis function (RBF). The cost parameter was set at 4 and the other parameters were fixed at the default values. The cost parameter used in the training process was selected from $\{0.5,1,2,4,6,8,10,12\}$. For the datasets used in this study, the RBF was found to provide the best results. In the random forest method without feature selection analysis, the number of trees was 10 and the number of features was 9 . In the random forest method with feature selection analysis, the number of trees was 10 and the number of features was 6 or 7 because the number of features selected by the feature selection method was small.

\section{Performance evaluation criteria}

The following measures were used to assess the performance of the classifiers used in this study: accuracy, sensitivity, F-measure, Matthew's correlation coefficient (MCC) $[71,72]$, and the area under the receiver operating characteristic curve (AUC) $[42,71]$. A trade-off between sensitivity and specificity was observed as the prediction threshold was varied. AUC is an effective means of comparing the overall prediction performance of different methods because it provides a single measure of overall threshold-independent accuracy. An AUC and MCC of 1 indicate perfect prediction accuracy. These measures are defined as follows:

$$
\begin{gathered}
\text { Accuracy }=\frac{T P+T N}{T P+F N+F P+T N} \\
\text { Sensitivity }=\frac{T P}{T P+F N} \\
\text { Specificity }=\frac{T N}{T N+F P} \\
F-\text { measure }=\frac{2 \times p r e c i s i o n \times r e c a l l}{p r e c i s i o n+r e c a l l} \\
M C C=\frac{(T P \times T N)-(F P \times F N)}{\sqrt{(T P+F P)(T P+F N)(T N+F P)(T N+F N)}}
\end{gathered}
$$

where $T P$ is the number of true positives, $F P$ is the number of false positives, $T N$ is the number of true negatives, $F N$ is the number of false negatives, and recall is equivalent to the sensitivity $[21,73]$. The formula for the AUC of a classifier is as follows:

$$
\hat{A}=\frac{S_{0}-n_{0}\left(n_{0}+1\right) / 2}{n_{0} n_{1}}
$$

where $S_{0}=\sum r_{i}, r_{i}$ is the rank of the $i$ th positive sample in the ranked list, $n_{0}$ is the number of positive samples, and $n_{1}$ is the number of negative samples $[74,75]$.

\section{Results and discussion}

\section{Performance of the four classification methods}

One of the goals of our experiment was to find a more discriminative and smaller feature set for specific function prediction, based solely on sequence-based features. Therefore, we initially gathered numerous features solely from the protein sequence. The features that were redundant or irrelevant were then removed by feature selection. After feature selection, the remaining number of features was small, while the accuracy of function classification was greater than that of the full-feature set. The selected features and the selection rates for the traditional features and our new features are listed in Tables 3 and 4.

A summary of the performance of the four methods in classifying the 11 protein classes is provided in Table 5 and Figure 1. Among all the methods, SVM without feature selection (SVM_FF) required more model-building time and had the lowest performance. However, this method did obtain the highest accuracy in two of the blind tests, for translation and fibre proteins ( 2 of the 11 protein classes).

SVM with feature selection (SVM_CFS) slightly outperformed the random forest method with and without feature selection (RF_CFS and RF_FF, respectively) and significantly outperformed SVM_FF. Given that more than one method had equal accuracy for some classes, the SVM_CFS method had the highest accuracy for classifying transcription, acetylcholine receptor inhibitor, G-protein coupled receptor, guanine nucleotide-releasing factor, fibre, and transmembrane proteins ( 6 of the 11 protein classes).

The random forest method without feature selection (RF_FF) had the highest accuracy for the following blind test sets: amino acid biosynthesis, acetylcholine receptor inhibitor, guanine nucleotide-releasing factor, fibre, and transmembrane proteins ( 5 of the 11 protein classes).

The random forest method with feature selection (RF_CFS) had the highest accuracy for classifying trans- 


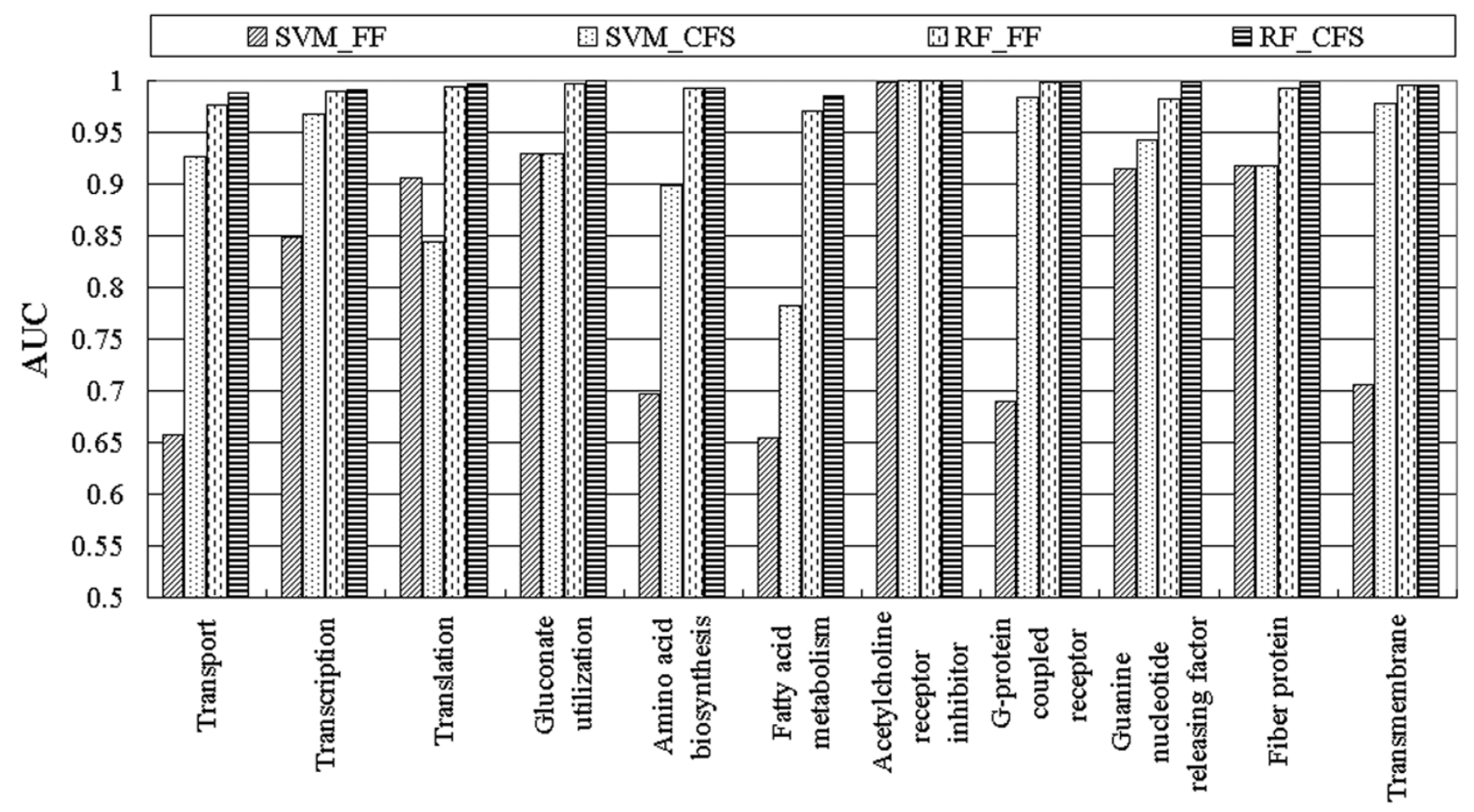

Protein Class

Figure I

Area under the ROC curves for the four methods for each protein class.

port, gluconate utilisation, amino acid biosynthesis, fatty acid metabolism, and acetylcholine receptor inhibitor proteins ( 5 of the 11 protein classes). Although both the RF_FF and RF_CFS methods had the highest accuracy for five protein classes, the performance of the RF_CFS method was better than that of the RF_FF method in terms of cost-effectiveness because a reduced-dimensional model was produced.

After careful analysis of the selected feature subsets and their performance in these experiments, the use of feature selection was found to improve classifier performance, as indicated in Table 5 and Figure 1. When comparing the SVM_FF and SVM_CFS methods, translation was the only protein class for which the SVM_FF method (AUC of 0.906, accuracy of 98.67) performed better than the SVM_CFS method (AUC of 0.844, accuracy of 97.78). For all the other classifications, the use of feature selection improved classifier performance. For classification of transport, transcription, amino acid biosynthesis, G-protein coupled receptor, and transmembrane proteins, both accuracy levels and AUCs significantly improved when feature selection was used. For example, the accuracy of transmembrane protein classification improved (79.81 to 97.84 ) and the AUC increased (0.706 to 0.978). In addi- tion, the number of features used for classification was 35 of 484. For the G-protein coupled receptor, 39 of 484 features were included, and the accuracy of the classification was dramatically improved by feature selection (77.07 to 98.17), as was the AUC (0.69 to 0.984).

Although the accuracy of the random forest method was not significantly improved by feature selection, the AUC value for RF_CFS was slightly higher than that for RF_FF, except for the amino acid biosynthesis, acetylcholine receptor inhibitor, and G-protein coupled receptor classes. The larger the AUC, the better is the performance of the model. By comparing the AUCs averaged over all 11 protein classes, the RF_CFS method was found to outperform the other methods (i.e. 0.995). Therefore, applying CFS to the dataset yielded improved performance and a more compact set of features.

For a more detailed evaluation of all the methods, several performance measures were applied. Detailed results for each method are presented in Tables $6,7,8$, and 9, with a focus on sensitivity, specificity, F-measure, and MCC. The consistent performance of each method for predicting the protein functions of the 11 protein classes in both the 10fold cross-validation test and the blind test demonstrates 
Table 6: Detailed results of SVM without feature selection (SVM_FF)

\begin{tabular}{|c|c|c|c|c|}
\hline Protein class & Sensitivity & Specificity & F-measure & MCC \\
\hline Transport & 31.54 & 100 & 0.48 & 0.46 \\
\hline Transcription & 71.08 & 98.81 & 0.83 & 0.73 \\
\hline Translation & 81.25 & 100 & 0.90 & 0.9 \\
\hline Gluconate utilisation & 85.71 & 100 & 0.92 & 0.92 \\
\hline Amino acid biosynthesis & 39.45 & 100 & 0.57 & 0.53 \\
\hline Fatty acid metabolism & 30.86 & 100 & 0.47 & 0.52 \\
\hline Acetylcholine receptor inhibitor & 100 & 99.51 & 0.95 & 0.95 \\
\hline G-protein coupled receptor & 38.02 & 100 & 0.55 & 0.53 \\
\hline Guanine nucleotide-releasing factor & 82.86 & 100 & 0.91 & 0.9 \\
\hline Fibre protein & 83.33 & 100 & 0.91 & 0.91 \\
\hline Transmembrane & 41.26 & 100 & 0.58 & 0.56 \\
\hline
\end{tabular}

MCC: Matthew's correlation coefficient

the validity of our methods: SVM_FF, \pm 3.0; SVM_CFS, \pm $2.1 ; \mathrm{RF} \_\mathrm{FF} \pm 1.8$; and RF_CFS \pm 1.2 ( \pm refers to the difference in accuracy between the training step and the blind test step). These results indicate that our models have good predictive power in discriminative testing processes.

Although good performance with the proposed new features was obtained using feature selection, we performed an additional experiment to demonstrate the usefulness of the proposed features in a clear and simple way, without relying on feature selection. The additional experiments were carried out using the 451 traditional features versus the 33 proposed features under the same conditions as the above experiments, and the performance of classification was compared (Tables 10 and 11). In the performance comparison with SVM, classification using only the 33 proposed features outperformed the 451 traditional features for 5 of the 11 protein classes (transport, amino acid biosynthesis, fatty acid metabolism, G-protein coupled receptor, and transmembrane). In the performance comparison with the random forest method, classification using only the 33 proposed features was superior or equal to use of the 451 traditional features for 4 of the 11 protein classes (translation, gluconate utilisation, fatty acid metabolism, and acetylcholine receptor inhibitor).

\section{Meaningful features for protein function prediction}

The 451 traditional features used for prediction of protein function have been described in previous reports $[17,22$ $24,28,32,36,37,43-45,76-78]$. The present study introduces new features based on negatively and positively charged residues and analyses their utility. The average number of new features selected by CFS was 5.4 for the 11 protein classes. The raw dataset was analysed for the selected features, and three examples are provided in Figures 2,3 , and 4 .

Figure 2 clearly demonstrates the differences in the means and standard deviations of the nine features used to classify guanine nucleotide-releasing factor (the opaque colour at the base of the bar graphs indicates the standard deviation). PPR and NNR for guanine nucleotide-releasing factor were higher than for the negative samples. For example, the NNR for guanine nucleotide-releasing factor was 7.04 (mean) \pm 1.72 (standard deviation), while the NNR for the negative samples was $3.79 \pm 2.0$. It is worth

Table 7: Detailed results of SVM with feature selection (SVM_CFS)

\begin{tabular}{|c|c|c|c|c|}
\hline Protein class & Sensitivity & Specificity & F-measure & $\mathrm{MCC}$ \\
\hline Transport & 87.58 & 97.83 & 0.92 & 0.87 \\
\hline Transcription & 98.31 & 95.01 & 0.97 & 0.93 \\
\hline Translation & 68.75 & 100 & 0.82 & 0.82 \\
\hline Gluconate utilisation & 85.71 & 100 & 0.92 & 0.92 \\
\hline Amino acid biosynthesis & 79.58 & 100 & 0.89 & 0.84 \\
\hline Fatty acid metabolism & 56.79 & 99.73 & 0.72 & 0.71 \\
\hline Acetylcholine receptor inhibitor & 100 & 100 & 1.00 & 1.00 \\
\hline G-protein coupled receptor & 99.24 & 97.54 & 0.98 & 0.96 \\
\hline Guanine nucleotide-releasing factor & 88.57 & 99.78 & 0.93 & 0.92 \\
\hline Fibre protein & 83.33 & 100 & 0.91 & 0.91 \\
\hline Transmembrane & 97.76 & 97.89 & 0.97 & 0.95 \\
\hline
\end{tabular}

MCC: Matthew's correlation coefficient 
Table 8: Detailed results of the random forest method without feature selection (RF_FF)

\begin{tabular}{|c|c|c|c|c|}
\hline Protein class & Sensitivity & Specificity & F-measure & MCC \\
\hline Transport & 87.58 & 95.89 & 0.84 & 0.91 \\
\hline Transcription & 96.87 & 92.4 & 0.89 & 0.95 \\
\hline Translation & 56.25 & 100 & 0.74 & 0.72 \\
\hline Gluconate utilisation & 85.71 & 100 & 0.92 & 0.92 \\
\hline Amino acid biosynthesis & 96.89 & 95.65 & 0.92 & 0.95 \\
\hline Fatty acid metabolism & 71.6 & 100 & 0.82 & 0.84 \\
\hline Acetylcholine receptor inhibitor & 100 & 100 & 1.00 & 1.00 \\
\hline G-protein coupled receptor & 95.44 & 99.11 & 0.95 & 0.97 \\
\hline Guanine nucleotide-releasing factor & 85.71 & 100 & 0.92 & 0.92 \\
\hline Fibre protein & 83.33 & 100 & 0.91 & 0.91 \\
\hline Transmembrane & 95.07 & 99.3 & 0.95 & 0.97 \\
\hline
\end{tabular}

MCC: Matthew's correlation coefficient

noting that negatively charged residues appear more frequently in the guanine nucleotide-releasing factor sequence than in those of other proteins. Furthermore, the NNR and PPR features are related to the number or percentage of negatively and positively charged residues, as these features were computed using a method based on charged residues. Because of this relationship, the mean percentages of positively charged residues and negatively charged residues were found to be similar to the PPR and NNR values, respectively. If the PPR for a specific protein family was high compared to that for other families, then the number of positively charged residues in that protein family was also higher than that in other families; similarly, if the NNR for a specific protein family was low, then the number of negatively charged residues in that protein family was also low. However, if the percentage of negatively charged residues was high and the NNR value was low, it is possible to infer that both negatively and positively charged residues are present in the sequences, because NNR and PPR provide information on whether the two charged residue types co-exist in the sequence. The greater the difference between the percent negatively charged residues and the NNR, the more frequent is the alternating occurrences of negatively and positively charged residues. For instance, golgi transport protein 1 [Swiss-Prot:Q9USJ2] consists of 129 amino acids with five negatively charged residues and eight positively charged residues. However, NNR and PPR were 0 and 2.32, respectively. This indicates that although the protein includes five negatively charged residues, the positively and negatively charged residues in the sequence always alternate among the neutral residues.

Previous studies in this field have analysed only the number or percentage of positively and negatively charged residues; however, the positions or regions of the charged residues in the sequence are very important in determining protein function and structure [79-82]. For example, Verma et al. [79] analysed a large panel of plaque-purified recovered viruses and demonstrated that the negatively charged residues at positions 440 and 441 were key residues that appeared to be involved in virus assembly. Therefore, although the total number of positively and negatively charged residues is important, residues in specific positions or local regions of the sequences are also important. Dist $(x, y)$ for PPR, NNR, and PNPR pro-

Table 9: Detailed results of the random forest method with feature selection (RF_CFS)

\begin{tabular}{|c|c|c|c|c|}
\hline Protein class & Sensitivity & Specificity & F-measure & $\mathrm{MCC}$ \\
\hline Transport & 90.27 & 97.10 & 0.93 & 0.88 \\
\hline Transcription & 96.63 & 92.87 & 0.95 & 0.90 \\
\hline Translation & 62.50 & 100.00 & 0.77 & 0.78 \\
\hline Gluconate utilisation & 100.00 & 100.00 & 1.00 & 1.00 \\
\hline Amino acid biosynthesis & 95.85 & 96.30 & 0.95 & 0.92 \\
\hline Fatty acid metabolism & 77.78 & 99.73 & 0.87 & 0.85 \\
\hline Acetylcholine receptor inhibitor & 100.00 & 100.00 & 1.00 & 1.00 \\
\hline G-protein coupled receptor & 96.58 & 97.99 & 0.97 & 0.95 \\
\hline Guanine nucleotide-releasing factor & 85.71 & 99.55 & 0.90 & 0.89 \\
\hline Fibre protein & 66.67 & 100.00 & 0.80 & 0.81 \\
\hline Transmembrane & 94.62 & 98.83 & 0.96 & 0.94 \\
\hline
\end{tabular}

MCC: Matthew's correlation coefficient 
Table 10: Comparative performance of the novel feature set and traditional feature set using SVM

\begin{tabular}{|c|c|c|c|c|c|c|c|c|c|c|}
\hline \multirow[t]{2}{*}{ Protein class } & \multicolumn{5}{|c|}{ Novel feature set (33 features) } & \multicolumn{5}{|c|}{ Traditional feature set ( $45 \mathrm{I}$ features) } \\
\hline & $\begin{array}{l}\text { Training } \\
\text { Accuracy }\end{array}$ & $\begin{array}{c}\text { Test } \\
\text { accuracy }\end{array}$ & Sensitivity & Specificity & AUC & $\begin{array}{l}\text { Training } \\
\text { accuracy }\end{array}$ & Test accuracy & Sensitivity & Specificity & $A \cup C$ \\
\hline Transport & 75.0273 & 73.31 & 36.2 & 100 & 0.681 & 73.2636 & 72.19 & 33.6 & 100 & 0.668 \\
\hline Transcription & 87.9723 & 88.15 & 99.3 & 77.2 & 0.882 & 92.5625 & 97.36 & 98.3 & 96.4 & 0.974 \\
\hline Translation & 97.0864 & 97.34 & 62.5 & 100 & 0.813 & 98.8642 & 98.67 & 81.3 & 100 & 0.906 \\
\hline $\begin{array}{l}\text { Gluconate } \\
\text { utilisation }\end{array}$ & 96.8288 & 96.22 & 71.4 & 100 & 0.857 & 98.7315 & 98.11 & 85.7 & 100 & 0.929 \\
\hline $\begin{array}{l}\text { Amino acid } \\
\text { biosynthesis }\end{array}$ & 74.8627 & 77.83 & 42.6 & 100 & 0.713 & 73.5272 & 77.43 & 41.5 & 100 & 0.708 \\
\hline $\begin{array}{l}\text { Fatty acid } \\
\text { metabolism }\end{array}$ & 92.4123 & 90.22 & 45.7 & 100 & 0.728 & 90.5586 & 87.77 & 32.1 & 100 & 0.66 \\
\hline $\begin{array}{l}\text { Acetylcholine } \\
\text { receptor } \\
\text { inhibitor }\end{array}$ & 98.448 & 99.06 & 80 & 100 & 0.9 & 100 & 99.53 & 100 & 99.5 & 0.998 \\
\hline $\begin{array}{l}\text { G-protein } \\
\text { coupled } \\
\text { receptor }\end{array}$ & 78.6998 & 80.87 & 48.3 & 100 & $0.74 I$ & 76.1838 & 77.35 & 38.8 & 100 & 0.694 \\
\hline $\begin{array}{l}\text { Guanine } \\
\text { nucleotide- } \\
\text { releasing factor }\end{array}$ & 97.4359 & 97.92 & 77.1 & 99.6 & 0.883 & 98.8681 & 98.75 & 82.9 & 100 & 0.914 \\
\hline Fibre protein & 96.789 & 95.89 & 0 & 100 & 0.5 & $99.847 \mid$ & 99.31 & 83.3 & 100 & 0.917 \\
\hline Transmembrane & 85.2931 & 85.67 & 58.3 & 100 & 0.791 & 79.8937 & 80.58 & 43.5 & 100 & 0.717 \\
\hline
\end{tabular}

AUC: Area under the curve.

Table I I: Comparative performance of the novel feature set and traditional feature set using the random forest

\begin{tabular}{|c|c|c|c|c|c|c|c|c|c|c|}
\hline \multirow[t]{2}{*}{ Protein class } & \multicolumn{5}{|c|}{ Novel feature set (33 features) } & \multicolumn{5}{|c|}{ Traditional feature set ( $45 \mathrm{I}$ features) } \\
\hline & $\begin{array}{l}\text { Training } \\
\text { accuracy }\end{array}$ & $\begin{array}{c}\text { Test } \\
\text { accuracy }\end{array}$ & Sensitivity & Specificity & $A \cup C$ & $\begin{array}{l}\text { Training } \\
\text { accuracy }\end{array}$ & Test accuracy & Sensitivity & Specificity & $A \cup C$ \\
\hline Transport & 91.3688 & 90.30 & 86.6 & 93 & 0.968 & 92.9764 & 93.39 & 89.9 & 95.9 & 0.975 \\
\hline Transcription & 90.9659 & 91.26 & 93.7 & 88.8 & 0.98 & 94.4252 & 95.33 & 96.4 & 94.3 & 0.99 \\
\hline Translation & 97.679 & 97.78 & 68.8 & 100 & 0.95 & 98.0741 & 97.78 & 68.8 & 100 & 0.996 \\
\hline $\begin{array}{l}\text { Gluconate } \\
\text { utilisation }\end{array}$ & 96.4059 & 98.11 & 85.7 & 100 & 0.997 & 97.2516 & 98.11 & 85.7 & 100 & 0.992 \\
\hline $\begin{array}{l}\text { Amino acid } \\
\text { biosynthesis }\end{array}$ & 93.7676 & 94.52 & 91.7 & 96.3 & 0.983 & 94.836 & 95.46 & 94.8 & 95.9 & 0.991 \\
\hline $\begin{array}{l}\text { Fatty acid } \\
\text { metabolism }\end{array}$ & 95.7242 & 94.44 & 72.8 & 99.2 & 0.97 & 96.2926 & 94 & 69.1 & 99.5 & 0.964 \\
\hline $\begin{array}{l}\text { Acetylcholine } \\
\text { receptor } \\
\text { inhibitor }\end{array}$ & 99.6896 & 100 & 100 & 100 & I & 99.8965 & 100 & 100 & 100 & I \\
\hline $\begin{array}{l}\text { G-protein } \\
\text { coupled } \\
\text { receptor }\end{array}$ & 94.4679 & 95.92 & 94.3 & 96.9 & 0.991 & 96.8745 & 97.18 & 94.7 & 98.7 & 0.993 \\
\hline $\begin{array}{l}\text { Guanine } \\
\text { nucleotide- } \\
\text { releasing factor }\end{array}$ & 96.7429 & 96.67 & 62.9 & 99.3 & 0.956 & 98.4985 & 97.92 & 74.3 & 99.8 & 0.992 \\
\hline Fibre protein & 97.4771 & 95.89 & 33.3 & 98.6 & 0.798 & 99.2355 & 99.31 & 83.3 & 100 & 0.998 \\
\hline Transmembrane & 93.555 & 93.52 & 87.4 & 96.7 & 0.978 & 95.9719 & 97.53 & 94.2 & 99.3 & 0.995 \\
\hline
\end{tabular}

AUC: Area under the curve. 


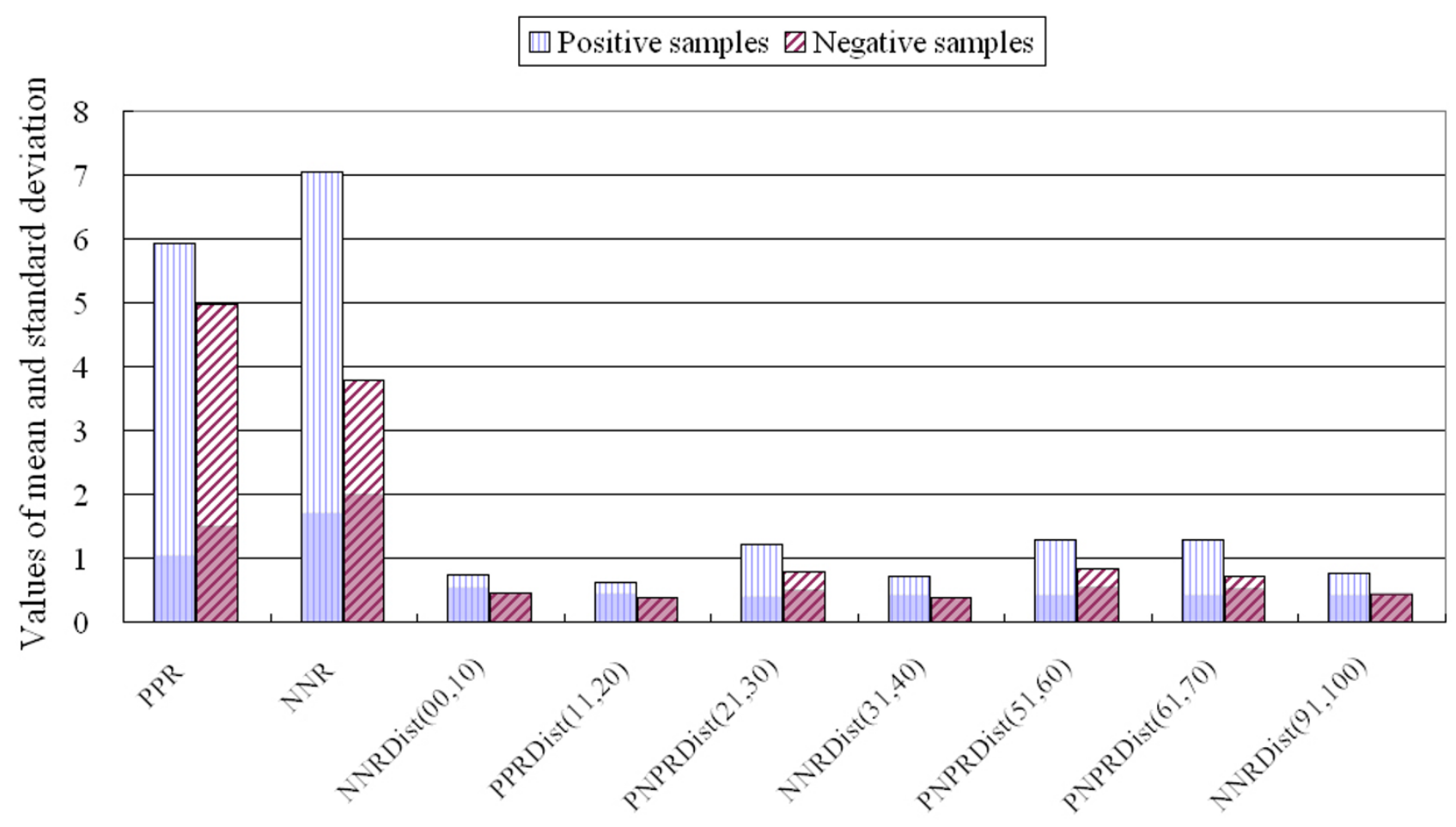

Features

Figure 2

Comparison of nine features used for classification of guanine nucleotide-releasing factor versus negative proteins.

vides information on negatively and positively charged residues in local regions of the sequence. Seven features that provide local information were selected for classification of the guanine nucleotide-releasing factor. For example, PNPRDist ${ }_{(61,70)}$ was $1.29 \pm 0.42$ for the positive classification samples and $0.72 \pm 0.53$ for the negative samples. These findings indicate that alternating positively and negatively charged residues occur more frequently in the local region from $61 \%$ to $70 \%$ in the guanine nucleotide-releasing factor sequence than in the negative protein samples. Therefore, local information on the distribution of negatively and positively charged residues in the interval was informative. Because of these essential differences, guanine nucleotide-releasing factor proteins can be predicted with a high level of accuracy.

Figure 3 presents the results of analysis of the raw data for two features used in classifying transcription proteins. PNPR for the positive classification samples was $12.04 \pm$ 3.56, while for the negative samples, PNPR was $7.84 \pm$ 3.03. These results indicate that continuous changes from a positively charged residue to the next negatively charged residue or vice versa occurred more frequently over the full sequence of transcription proteins than in the negative samples.

Figure 4 presents the results of analysis of the raw dataset for four features, when the proteins were classified using the random forest method. The mean values of the four selected features for gluconate utilisation were significantly lower than those of the negative samples. For example, PNPRDist $t_{(11,20)}$ for the gluconate utilisation sequences was $1.03 \pm 0.3$, while PNPRDist $t_{(11,20)}$ for the negative samples was $1.3 \pm 0.6$. These results indicate that there were fewer continuous changes from a positively charged residue to the next negatively charged residue or vice versa in the local region from $11 \%$ to $20 \%$ of the gluconate utilisation sequences than in the negative samples.

There are two ways of using positively charged residues in classification and prediction of protein function. One process uses the positively charged residues arginine $(\mathrm{R})$, histidine $(\mathrm{H})$, and lysine $(\mathrm{K})[20]$, while the other uses only arginine (R) and lysine (K) [24]. Although defining two groups of positively charged residues is potentially useful, we found that the use of arginine, histidine, and 


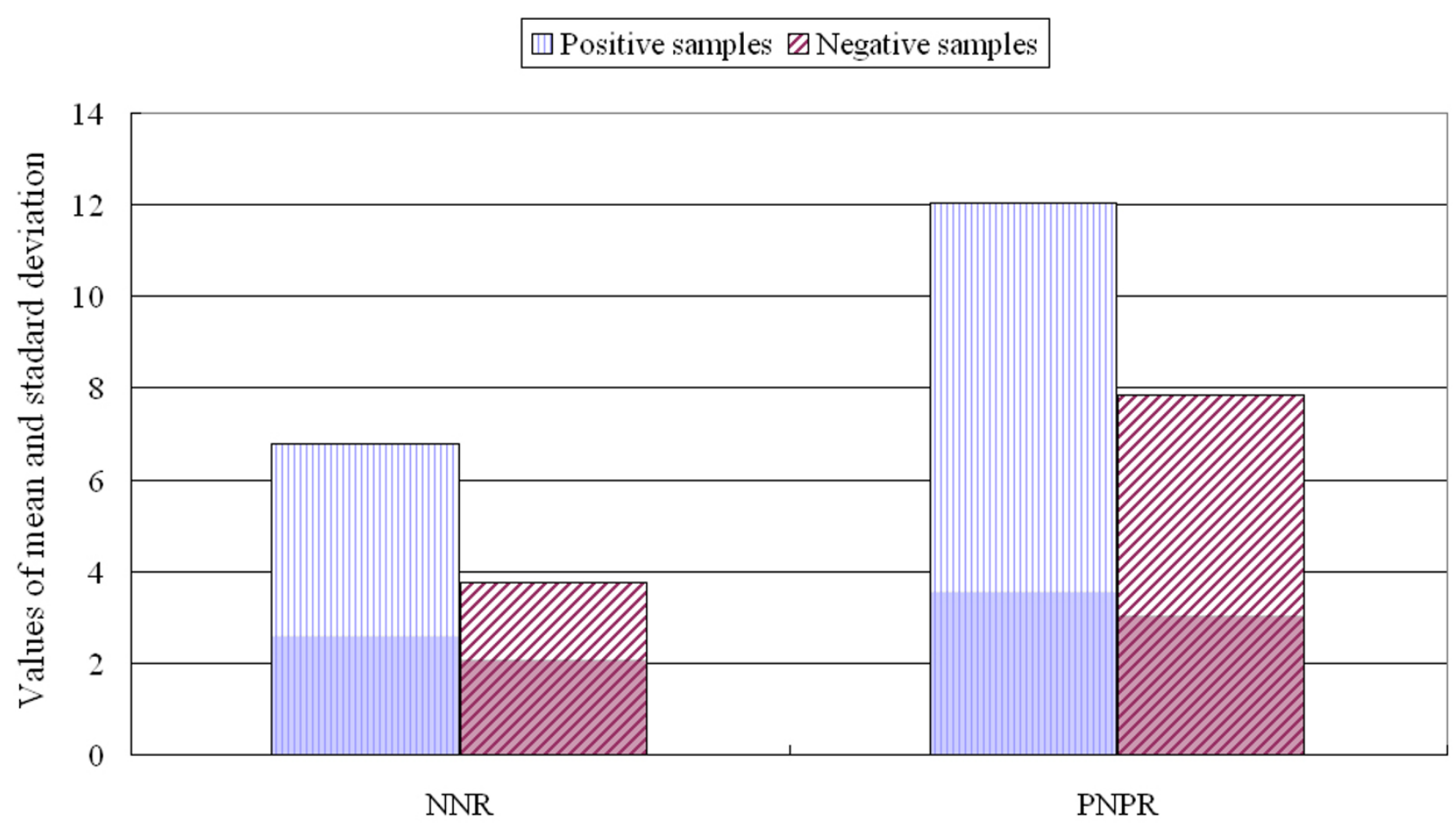

Features

Figure 3

Comparison of three features used for classification of transcription versus negative proteins.

lysine achieved better results than did arginine and lysine. Specifically, the former $(\mathrm{R}, \mathrm{H}$, and $\mathrm{K})$ was useful in classifying gluconate utilisation, fatty acid metabolism, G-protein coupled receptor, transcription, and transport proteins, while the latter ( $\mathrm{R}$ and $\mathrm{K}$ ) was useful only in classifying fibre proteins.

\section{Significant findings}

The following is a summary of the benchmark comparisons and important findings of this study for prediction of protein function over a broad range of cellular components, molecular functions, and biological processes. Analyses were conducted by SVM and the random forest method with and without feature selection, based on many traditional and proposed features extracted from the sequences.

- Using a larger number of features to predict protein function does not always result in improved performance. In terms of accuracy and AUC, SVM with feature selection has distinct performance advantages with this type of data, indicating that removal of the many redundant and irrelevant features by feature selection can improve pre- diction performance. However, there was no significant difference in prediction performance for the random forest method with and without feature selection.

- The use of a particular classifier does not always result in improved performance. There is no single method that is optimal for all conditions because the performance of a method depends on the type of data involved, the size of the dataset, the number of features involved, the type of extracted features, and whether feature selection is used, among other things. Therefore, selection of the optimal classifier for a given dataset depends on an understanding of machine-learning algorithms, feature selection processes, and biological background information relevant to the dataset.

- Features useful for predicting a specific protein function in a given dataset are not always useful for predicting another protein function - discriminative and informative features differ according to protein function. Therefore, identifying discriminative features applicable to a broad range of protein classes is difficult. 


\section{Positive samples $\square$ Negative samples}

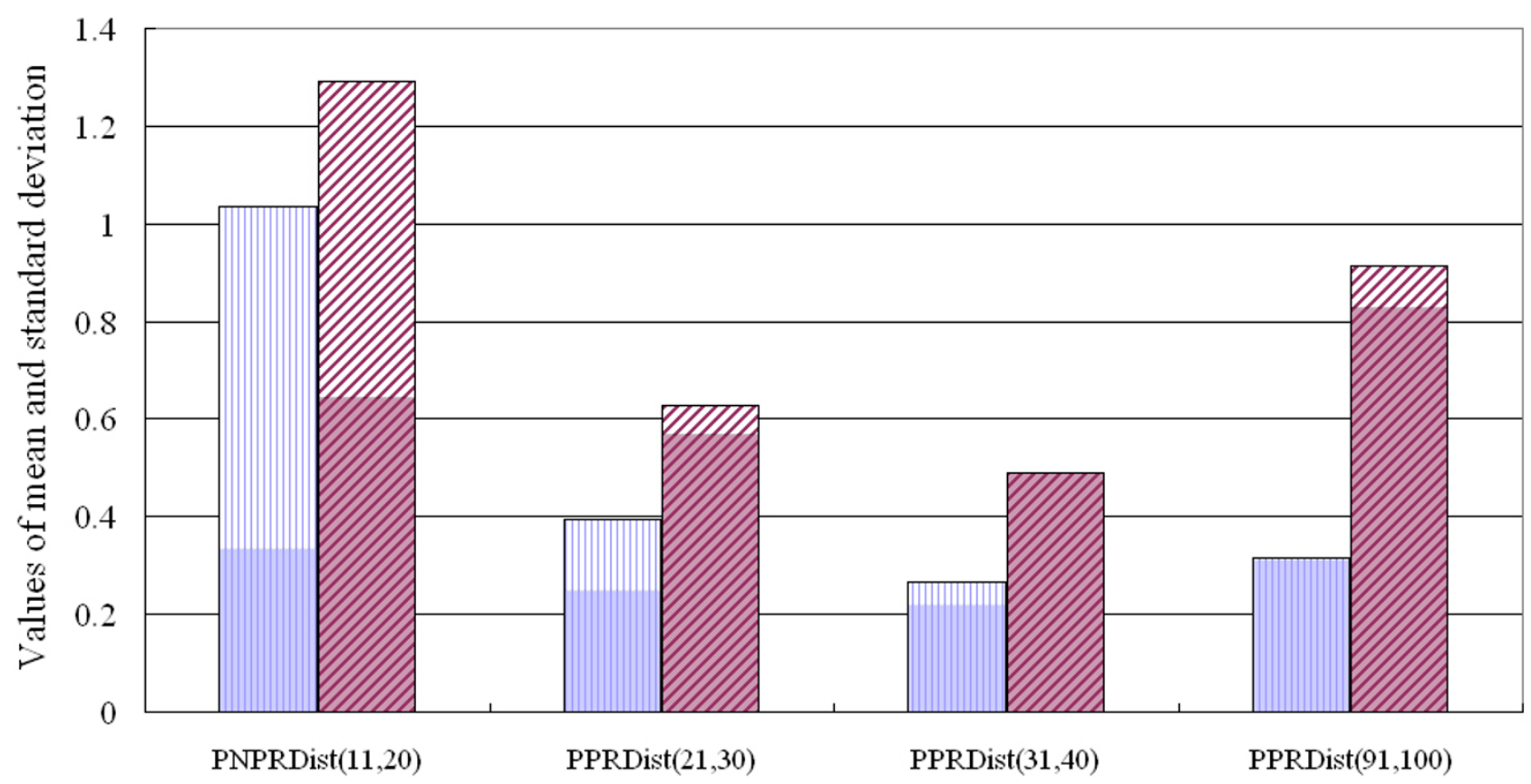

Features

Figure 4

Comparison of local information used for classification of gluconate utilisation versus negative proteins.

- Although many methods have recently been proposed for predicting protein function, most methods are not suitable for function prediction under high-throughput conditions, because they require information on protein structure. Currently, there is much more data available on protein sequences than on protein structures; thus, the methods developed in this study focused on predicting protein function based solely on features extracted from the protein sequence. This reduces the effort required to extract useful features, as the predictive or experimental work required to acquire structural information is both costly and time-consuming. In the experiments undertaken in this study, we found that sequence-based classifiers can also generate very good results.

- Local information regarding the protein sequence is meaningful in predicting protein function; several examples have been presented to demonstrate its usefulness. Although identifying local information for a sequence is difficult and the information does not always correspond to striking difference in protein function, unique features extracted from specific positions or local regions can be predicted with a high level of accuracy.
- The numbers or percentages of positively and negatively charged residues are some of the most important and well-known features used for function prediction. $P P R$ and NNR were extracted from sequences based on the presence of negatively and positively charged residues. These novel features include information on the existence of negatively and positively charged residues as well as the manner in which the two charged residue types co-exist in a sequence. $P P R$ and NNR were found to be selected more frequently for function prediction than were the number of negatively and positively charged residues. Thus, these features appear to be highly correlated with protein class and have a low inter-correlation with each other.

The above results indicate that it is possible to generate accurate predictions for a broad range of protein functions without the use of sequence or structural similarities. Feature selection improves predictions for a variety of protein functions, but does not always ensure improved performance, depending on the dataset and the method used. Finally, local information for protein sequences is meaningful for predicting protein function, and a feature set with good performance and dimensional reduction was 
identified, as many features initially included in this study were removed by CFS.

\section{Conclusion}

Many previous studies have attempted to biologically and computationally determine meaningful and accurate features that assist in predicting protein function. Features that show an obvious propensity for predicting many different protein functions have not yet been reported, and this provides a motivation for discovering the relationship between features and protein function.

This paper described a highly accurate prediction method capable of identifying protein function by using features extracted solely from protein sequences, irrespective of sequence and structural similarities. In this study, the PPR, NNR, PNPR, and Dist $(x, y)$ features were introduced. In predicting the functions of 11 different proteins, a high performance $(94.23-100 \%)$ was achieved and predictive features for several protein classes were effectively identified.

The results presented here suggest that our new features, developed in the course of this study, will be useful in predicting many protein class functions. We believe that prediction performance can be improved by combining sequence-based features and additional features, such as predicted secondary structure, surface area, and subcellular location. Accordingly, further insight into feature analysis and biological understanding is needed. In future studies, we will apply this method to predict the functions of proteins that have not been identified by sequence alignment.

\section{Competing interests}

The authors declare that they have no competing interests.

\section{Authors' contributions}

BJL conducted the experiments and analysis, conceived the concepts of PPR, NNR, PNPR, and Dist $(x, y)$, and wrote the manuscript. MSS and YJO assisted in developing the method and revising the manuscript. KHR and HSO supervised the work, provided useful suggestions to improve performance, and revised the manuscript. All authors read and approved the manuscript.

\section{Acknowledgements}

This work was supported by a grant from the Korean Ministry of Education, Science, and Technology (The Regional Core Research Programme/Chungbuk BIT Research-Oriented University Consortium) and by Basic Science Research Program through the National Research Foundation of Korea (NRF)funded by the Ministry of Education, Science and Technology (MEST) (ROI-2007-000-10926-0, RI I-2008-0I4-02002-0).

\section{References}

I. Altschul SF, Gish W, Miller W, Myers EW, Lipman DJ: Basic local alignment search tool. J Mol Biol 1990, 21 5:403-4I0.

2. Altschul SF, Madden TL, Schaffer AA, Zhang J, Zhang Z, Miller W, Lipman DJ: Gapped BLAST and PSI-BLAST: a new generation of protein database search programs. Nucleic Acids Res 1997, 35:3389-3402.

3. Pearson WR, Lipman DJ: Improved tools for biological sequence comparison. Proc Natl Acad Sci USA 1988, 85:2444-2448.

4. Benner SA, Chamberlin SG, Liberles DA, Govindarajan S, Knecht L: Functional inferences from reconstructed evolutionary biology involving rectified databases - an evolutionarily grounded approach to functional genomics. Res Microbiol 2000, 151:97-106.

5. Cai CZ, Han LY, Ji ZL, Chen X, Chen YZ: Enzyme family classification by support vector machines. Proteins 2004, 55:66-76.

6. Dobson PD, Doig AJ: Predicting enzyme class from protein structure without alignments. J Mol Biol 2005, 345: I87-199.

7. Han LY, Cai CZ, Ji ZL, Cao ZW, Cui J, Chen YZ: Predicting functional family of novel enzymes irrespective of sequence similarity: a statistical learning approach. Nucleic Acids Res 2004, 32:6437-6444.

8. Wang X, Schroeder D, Dobbs D, Honavar V: Automated datadriven discovery of motif-based protein function classifiers. Inf Sci 2003, 155:1-18.

9. Lapinsh M, Gutcaits A, Prusis P, Post C, Lundstedt T, Wikberg JES: Classification of $G$-protein coupled receptors by alignmentindependent extraction of principal chemical properties of primary amino acid sequences. Protein Sci 2002, I I :795-805.

10. Rost B: Twilight zone of protein sequence alignments. Protein Eng 1999, 12:85-94.

II. Hobohm U, Sander C: A sequence property approach to searching protein databases. J Mol Biol 1995, 25 I:390-399.

12. Claeyssens M, Henrissat B: Specificity mapping of cellulolytic enzymes: classification into families of structurally related proteins confirmed by biochemical analysis. Protein Sci 1992, I:1293-1297.

13. Karp PD: What we do not know about sequence analysis and sequence database. Bioinformatics 1998, 14:753-754.

14. Hawkins T, Kihara D: Function prediction of uncharacterized proteins. J Bioinform Comput Biol 2007, 5: I-30.

15. Holm L, Sander C: Dali: a network tool for protein structure comparison. Trends Biochem Sci 1995, 20:478-480.

16. Kawabata T: MATRAS: a program for protein 3D structure comparison. Nucleic Acids Res 2003, 3 1:3367-3369.

17. Eidhammer I, Jonassen I, Taylor WR: Protein structure comparison and structure patterns. J Comput Biol 2000, 7:685-716.

18. Friedberg I: Automated protein function prediction-the genomic challenge. Brief Bioinformatics 2006, 7:225-242.

19. Russell RB, Saqi MA, Bates PA, Sayle RA, Sternberg MJ: Recognition of analogous and homologous protein folds - assessment of prediction success and associated alignment accuracy using empirical substitution matrices. Protein Eng 1998, I I:I-9.

20. Syed U, Yona G: Enzyme function prediction with interpretable models. In Computational Systems Biology Edited by: Samudrala R, McDermott J, Bumgarner R. New York: Humana Press; 2007: I-33.

21. Borro LC, Oliveira SRM, Yamagishi MEB, Mancini AL, Jardine JG, Mazoni I, Santos EHD, Higa RH, Kuser PR, Neshich G: Predicting enzyme class from protein structure using Bayesian classification. Genet Mol Res 2006, 5: 193-202.

22. Cai CZ, Han LY, Ji ZL, Chen X, Chen YZ: SVM-Prot: web-based support vector machine software for functional classification of a protein from its primary sequence. Nucleic Acids Res 2003, 31:3692-3697.

23. Jensen LJ, Gupta R, Blom N, Devos D, Tamames J, Kesmir C, Nielsen $\mathrm{H}$, Stærfeldt HH, Rapacki K, Workman C, Andersen CAF, Knudsen S, Krogh A, Valencia A, Brunak S: Prediction of human protein function from post-translational modifications and localization features. J Mol Biol 2002, 3 1 9: 1257-1265.

24. Jensen LJ, Skovgaard M, Brunak S: Prediction of novel archaeal enzymes from sequence-derived features. Protein Sci 2002, 3:2894-2898. 
25. Pasquier C, Promponas VJ, Hamodrakas SJ: PRED-CLASS: cascading neural networks for generalized protein classification and genome-wide applications. Proteins 200I, 44:36I-369.

26. Chen YC, Lin YS, Lin CJ, Hwang JK: Prediction of the bonding states of cysteines using the support vector machines based on multiple feature vectors and cysteine state sequences. Proteins 2004, 55: I036-1042.

27. Yuan Z, Burrage K, Mattick JS: Prediction of protein solvent accessibility using support vector machines. Proteins 2002, 48:566-570

28. Noble WS, Ben-Hur A: Integrating information for protein function prediction. In Bioinformatics-From Genomes to Therapies Edited by: Lengauer T. Weinheim: Wiley- $\mathrm{VCH}$ Verlag $\mathrm{GmbH}$ \& Co $\mathrm{KGaA} ; 2007: 1297-1314$

29. Cai CZ, Wang WL, Sun LZ, Chen YZ: Protein function classification via support vector machine approach. Math Biosci 2003, 185: III-I22.

30. Bendtsen JD, Jensen LJ, Blom N, Heijne GV, Brunak S: Featurebased prediction of non-classical and leaderless protein secretion. Protein Eng Des Sel 2004, I7:349-356.

31. Dobson PD, Doig AJ: Distinguishing enzyme structures from non-enzymes without alignments. J Mol Biol 2003, 330:77I-783.

32. Al-Shahib A, Breitling R, Gilbert D: FRANKSUM: new feature selection method for protein function prediction. Int J Neural Syst 2005, I 5:250-275.

33. Al-Shahib A, Breitling R, Gilbert D: Feature selection and the class imbalance problem in predicting protein function from sequence. Appl Bioinformatics 2005, 4:195-203.

34. Wang Y, Tetko IV, Hall MA, Frank E, Facius A, Mayer KFX, Mewes HW: Gene selection from microarray data for cancer classification - a machine learning approach. Comput Biol Chem 2005, 29:37-46.

35. Zhao XM, Li X, Chen L, Aihara K: Protein classification with imbalanced data. Proteins 2008, 70: I | 25- II 32

36. Ding $\mathrm{CH}$, Dubchak I: Multi-class protein fold recognition using support vector machines and neural networks. Bioinformatics 200I, I 7:349-358.

37. Fujishima K, Komasa M, Kitamura S, Suzuki H, Tomita M, Kanai A: Proteome-wide prediction of novel DNA/RNA-binding proteins using amino acid composition and periodicity in the hyperthermophilic archaeon Pyrococcus furiosus. DNA Res 2007, 14:91-102

38. Borgwardt KM, Ong CS, Schonauer S, Vishwanathan SVN, Smola AJ, Kriegel HP: Protein function prediction via graph kernels. Bioinformatics 2005, 21 : il-il 0.

39. Zheng G, Qian Z, Yang Q, Wei C, Xie L, Zhu Y, Li Y: The combination approach of SVM and ECOC for powerful identification and classification of transcription factor. $B M C$ Bioinformatics 2008, 9:282.

40. Tung CW, Ho SY: Computational identification of ubiquitylation sites from protein sequences. BMC Bioinformatics 2008 9:310.

41. Judson R, Elloumi F, Setzer RW, Li Z, Shah I: A comparison of machine learning algorithms for chemical toxicity classification using a simulated multi-scale data model. BMC Bioinformatics 2008, 9:24I.

42. Garg A, Gupta D: VirulentPred: a SVM based prediction method for virulent proteins in bacterial pathogens. $B M C$ Bioinformatics 2008, 9:62.

43. Kumar M, Gromiha MM, Raghava GPS: Identification of DNAbinding proteins using support vector machines and evolutionary profiles. BMC Bioinformatics 2007, 8:463.

44. Pugalenthi G, Tang K, Suganthan PN, Archunan G, Sowdhamini R: A machine learning approach for the identification of odorant binding proteins from sequence-derived properties. BMC Bioinformatics 2007, 8:35I.

45. Li Q, Lai L: Prediction of potential drug targets based on simple sequence properties. BMC Bioinformatics 2007, 8:353.

46. Chen XW, Liu M: Prediction of protein-protein interactions using random decision forest framework. Bioinformatics 2005 , 21:4394-4400

47. Amaratunga D, Cabrera J, Lee YS: Enriched random forests. Bioinformatics 2008, 24:2010-2014.

48. Davies MN, Secker A, Freitas AA, Mendao M, Timmis J, Flower DR: On the hierarchical classification of $G$ protein-coupled receptors. Bioinformatics 2007, 23:3/I3-3/I8.
49. Halperin I, Glazer DS, Wu S, Altman RB: The FEATURE framework for protein function annotation: modelling new functions, improving performance, and extending to novel applications. BMC Genomics 2008, 16:9.

50. Gustafson AM, Snitkin ES, Parker SC], DeLisi C, Kasif S: Towards the identification of essential genes using targeted genome sequencing and comparative analysis. BMC Genomics 2006 , 7:265.

5I. Guan Y, Myers CL, Hess DC, Barutcuoglu Z, Caudy AA, Troyanskaya OG: Predicting gene function in a hierarchical context with an ensemble of classifiers. Genome Biol 2008, 9:S3.

52. Caragea C, Sinapov J, Silvescu A, Bobbs D, Honavar V: Glycosylation site prediction using ensembles of support vector machine classifiers. BMC Bioinformatics 2007, 8:438.

53. Ge G, Wong GW: Classification of premalignant pancreatic cancer mass-spectrometry data using decision tree ensembles. BMC Bioinformatics 2008, 9:275.

54. Strobl C, Boulesteix AL, Kneib T, Augustin T, Zeileis A: Conditional variable importance for random forests. BMC Bioinformatics 2008, 9:307.

55. Statnikov A, Wang L, Aliferis CF: A comprehensive comparison of random forests and support vector machines for microarray-based cancer classification. BMC Bioinformatics 2008, 9:319.

56. Todd $A E$, Orengo CA, Thornton JM: Evolution of function in protein superfamilies, from a structural perspective. J Mol Biol 2001, 307: III3-1143,

57. Bairoch A, Apweiler R: The SWISS-PROT protein sequence data bank and its supplement TrEMBL in 1999. Nucleic Acids Res 1999, 27:49-54.

58. Zdobnov EM, Lopez R, Apweiler R: The EBI SRS server - new features. Bioinformatics 2002, I 8: I I49-II50.

59. Gasteiger E, Hoogland C, Gattiker A, Duvaud S, Wilkins MR, Appel $R D$, Bairoch A: Protein identification and analysis tools on the ExPASy Server. In The Proteomics Protocols Handbook Edited by: John MW. New York: Humana Press; 2005:57I-607.

60. Truniger V, Lazaro JM, Esteban FJ, Blanco L, Salas M: A positively charged residue of $\phi 29$ DNA polymerase, highly conserved in DNA polymerases from families $A$ and $B$, is involved in binding the incoming nucleotide. Nucleic Acids Res 2002, 30: $1483-1492$.

61. Cappello V, Tramontano A, Koch U: Classification of proteins based on the properties of the ligand-binding site: the case of adenine-binding proteins. Proteins 2002, 47:106-II5.

62. Caruana R, de Sa VR: Benefitting from the variables that variable selection discards. J Mach Learn Res 2003, 3:1245-1264.

63. Saeys $Y$, Inza I, Larranaga P: A review of feature selection techniques in bioinformatics. Bioinformatics 2007, 23:2507-25I7.

64. Guyon I, Elisseeff A: An introduction to variable and feature selection. J Mach Learn Res 2003, 3: I I57-II 82.

65. Hall MA, Holmes B: Benchmarking attribute selection techniques for discrete class data mining. IEEE Trans Knowl Data Eng 2003, I5:I-16.

66. Fayyad U, Irani K: Multi-interval discretization of continuousvalued attributes for classification learning. In Proceedings of the 13th International Joint Conference on Al: 28 August-3 September 1993; Chambéry San Francisco: Morgan Kaufmann; 1993:1022-1027.

67. Ian H: Data Mining: Practical Machine Learning Tools and Techniques 2005 [http://www.cs.waikato.ac.nz/ml/weka/]. San Francisco: Morgan Kaufmann

68. Breiman L: Random forests. In Machine Learning Edited by: Schapire RE. Netherlands: Springer; 2001:5-32.

69. Chang CC, Lin CJ: LIBSVM: a library for support vector machines. 200I [http://www.csie.ntu.edu.tw/ cjlin/libsvm].

70. El-Manzalawy Y: WLSVM. 2005 [http://www.cs.iastate.edu/ - yasser/wlsvm/]

71. Baldi P, Brunak S, Chauvin Y, Andersen CAF, Nielsen H: Assessing the accuracy of prediction algorithms for classification: an overview. Bioinformatics 2000, 16:412-424.

72. Matthews BW: Comparison of the predicted and observed secondary structure of T4 phage lysozyme. Biochim Biophys Acta 1975, 405:442-45।.

73. Tan PN, Steinbach M, Kumar V: Introduction to Data Mining Boston: Addison Wesley; 2006.

74. Huang J, Ling CX: Using AUC and accuracy in evaluating learning algorithms. IEEE Trans Knowl Data Eng 2005, 17:299-3I0. 
75. Hand DJ, Till RJ: A simple generalisation of the area under the roc curve for multiple class classification problems. In Machine Learning Edited by: Aha DW. Netherlands: Kluwer Academic Publishers; 200I:I7I-186.

76. Ofran $\mathrm{Y}$, Margalit $\mathrm{H}$ : Proteins of the same fold and unrelated sequences have similar amino acid composition. Proteins 2006, 64:275-279.

77. Xie $D$, Li A, Wang M, Fan Z, Feng H: LOCSVMPSI: a web server for subcellular localization of eukaryotic proteins using SVM and profile of PSI-BLAST. Nucleic Acids Res 2005, 33:WI 05-I I0.

78. Davies MN, Secker A, Freitas AA, Clark E, Timmis J, Flower DR: Optimizing amino acid groupings for GPCR classification. Bioinformatics 2008, 24:1980-1986.

79. Verma S, Bednar V, Blount A, Hogue BG: Identification of functionally important negatively charged residues in the carboxy end of mouse hepatitis coronavirus A59 nucleocapsid protein. J Virol 2006, 80:4344-4355.

80. Andersson H, Bakker E, Heijne G: Different positively charged amino acids have similar effects on the topology of a polytopic transmembrane protein in Escherichia coli. J Biol Chem 1992, 267:|49|-|495.

8I. Rutz C, Rosenthal W, Schulein R: A single negatively charged residue affects the orientation of a membrane protein in the inner membrane of Escherichia coli only when it is located adjacent to a transmembrane domain. J Biol Chem 1999, 274:33757-33763.

82. Boyd D, Manoil C, Beckwith J: Determinants of membrane protein topology. Proc Natl Acad Sci USA 1987, 84:8525-8529.

Publish with Bio Med Central and every scientist can read your work free of charge

"BioMed Central will be the most significant development for disseminating the results of biomedical research in our lifetime. "

Sir Paul Nurse, Cancer Research UK

Your research papers will be:

- available free of charge to the entire biomedical community

- peer reviewed and published immediately upon acceptance

- cited in PubMed and archived on PubMed Central

- yours - you keep the copyright 